\title{
Genetic fingerprint defines hematopoietic stem cell pool size and function
}

\author{
Tatsuya Morishima ${ }^{1,2}$ and Hitoshi Takizawa ${ }^{1,3}$
}

${ }^{1}$ Laboratory of Stem Cell Stress, International Research Center for Medical Sciences, Kumamoto University; ${ }^{2}$ Laboratory of Hematopoietic Stem Cell Engineering, International Research Center for Medical Sciences, Kumamoto University and ${ }^{3}$ Center for Metabolic Regulation of Healthy Aging, Kumamoto University, Kumamoto, Japan

E-mail: HITOSHI TAKIZAWA - htakizawa@kumamoto-u.ac.jp or TATSUYA MORISHIMA - tatsuyam@kumamoto-u.ac.jp

doi:10.3324/haematol.2019.241299

$\mathrm{H}$ ematopoietic stem cells (HSC) are at the apex of the hematopoietic tree, with self-renewal and multilineage differentiation potential. On the one hand, HSC can replenish the mature blood cells by differentiating into lineage-committed progenitor cells in response to the shortage of blood cells under both homeostatic and stressed conditions, such as bleeding and infection. On the other hand, HSC replicate themselves to maintain their number. This differentiation and selfrenewal needs to be strictly regulated by gene expression regulation in order to maintain life-long hematopoiesis. ${ }^{1}$ Gene expression is in general regulated by "cis- and transregulatory elements". ${ }^{2}$ Trans-regulatory elements are defined as factors which regulate expression of distal genes (e.g. transcription factors), while cis-regulatory elements are defined as non-coding DNA sequences which regulate expression of proximal genes (e.g. promoter and enhancer regions). These cis-regulatory elements play important roles in evolution in which polymorphisms occur in cis-regulatory elements and contribute to phenotypic diversity of organisms within well-conserved genes. ${ }^{3}$ Epigenetics plays important roles in HSC regulation, as epigenetic dysregulation in HSC is a key driver for HSC aging and hematopoietic malignancies. ${ }^{4}$ Epigenetic regulation is also controlled by cis- and transregulatory elements. For example, histone modifications function as trans-regulatory elements, whereas DNA methylations function as cis-regulatory elements.

Phenotypic diversity is frequently caused by genetic variations such as single nucleotide polymorphism (SNP). It was reported that the size and function of the HSC pool vary between mice strains, ${ }^{5.9}$ which suggests genetic background, such as SNP and copy number variations, define HSC homeostasis. In 2007, Liang et al. identified latexin $(L x n)$ as an HSC regulatory gene whose expression level is inversely correlated with HSC number. ${ }^{10}$ Although a variation in $L x n$ gene expression and HSC number in different tested mouse strains was shown, the mechanism underlying regulation of $L x n$ gene expression and its variation between mice strains remained unknown.

In this issue of Haematologica, the same group who published the above-mentioned paper, ${ }^{10}$ Zhang et al. identified the promoter region of the Lxn gene that controls the level of $L x n$ gene expression via both HMGB2, a chromatin protein, and genetic variations in the promoter region. ${ }^{11}$ To study the transcriptional regulation of $L x n$, the authors characterized the upstream region of the $L x n$ gene. Based on the natural variation of Lxn expression, they searched SNP with CpG island and identified a region with strong promoter activity in the upstream. Subsequently, DNA pulldown in combination with mass spectrometry analysis were performed to identify pro- teins bound to this region, and HMGB2 was found to bind to the promoter region and to suppress $L x n$ gene promoter activity. To further confirm the regulatory role of HMGB2 in Lxn gene expression, Zhang et al. performed a gene knockdown experiment with EML cells, which share some of the characteristics of HSC..$^{12}$ This showed that HMGB2 knockdown suppresses EML cell growth and that additional $L x n$ gene knockdown could rescue this growth, suggesting that $L x n$ was one of the transcriptional targets of HMGB2. Consistent with the previous reports concerning the phenotype of cells overexpressing Lxn, ${ }^{13,14}$ the HMGB2 knockdown cells showed enhanced apoptosis and cell cycle arrest, which could in part be rescued by Lxn gene knockdown. These data suggest HMGB2 positively regulates HSC survival and proliferation by suppressing expression of $L x n$ and $L x n$-regulated apoptosis. Similar data were also shown in Lin:Sca- $1^{+} \mathrm{c}-$ $\mathrm{Kit}^{+}$(LSK) cells primarily isolated from mouse bone marrow that contain HSC. HMGB2 knockdown in LSK cells showed suppressed proliferation, enhanced apoptosis and cell cycle arrest in vitro. In transplantation experiments, HMGB2 knockdown in LSK cells showed decreased reconstitution of whole peripheral blood cells and bone marrow LSK cells and long-term HSC in transplant recipients, indicating that HMGB2 plays an important role in HSC function in vivo.

The previous finding that $L x n$ expression level is correlated with HSC numbers ${ }^{10}$ led the authors to hypothesize that the SNP in the promoter region of the Lxn gene may contribute to a variation in Lxn expression and HSC number. To test this, the authors introduced $\mathrm{G}$ to $\mathrm{C}$ mutation in the HMGB2 binding sequence in the Lxn gene promoter region and found that the $\mathrm{G}$ allele showed higher promoter activity for $L x n$ expression compared to the C allele. Furthermore, when Lxn gene expression and bone marrow HSC number were analyzed in different mouse strains carrying the $\mathrm{G}$ or $\mathrm{C}$ allele in this SNP region, the mice strain carrying the $\mathrm{C}$ allele showed relatively lower Lxn protein expression and higher HSC number compared to those carrying the $\mathrm{G}$ allele. These data suggest that a genetic variant in the $L x n$ gene promoter region defines the variation in Lxn gene expression level and HSC number.

Together, Zhang et al. revealed that the transcription of Lxn regulating HSC function, at least as far as apoptosis is concerned, was controlled by both trans-regulatory element, HMGB2, and cis-regulatory element, as genetic variation was observed in the $L x n$ gene promoter region (Figure 1). HMGB2 is known as a chromatin-associated protein which remodels chromatin structure and gene expression. ${ }^{15}$ Although the molecular mechanism by which HMGB2 regulates $L x n$ gene transcription remains unclear, all the data provided in this study suggest $L x n$ 


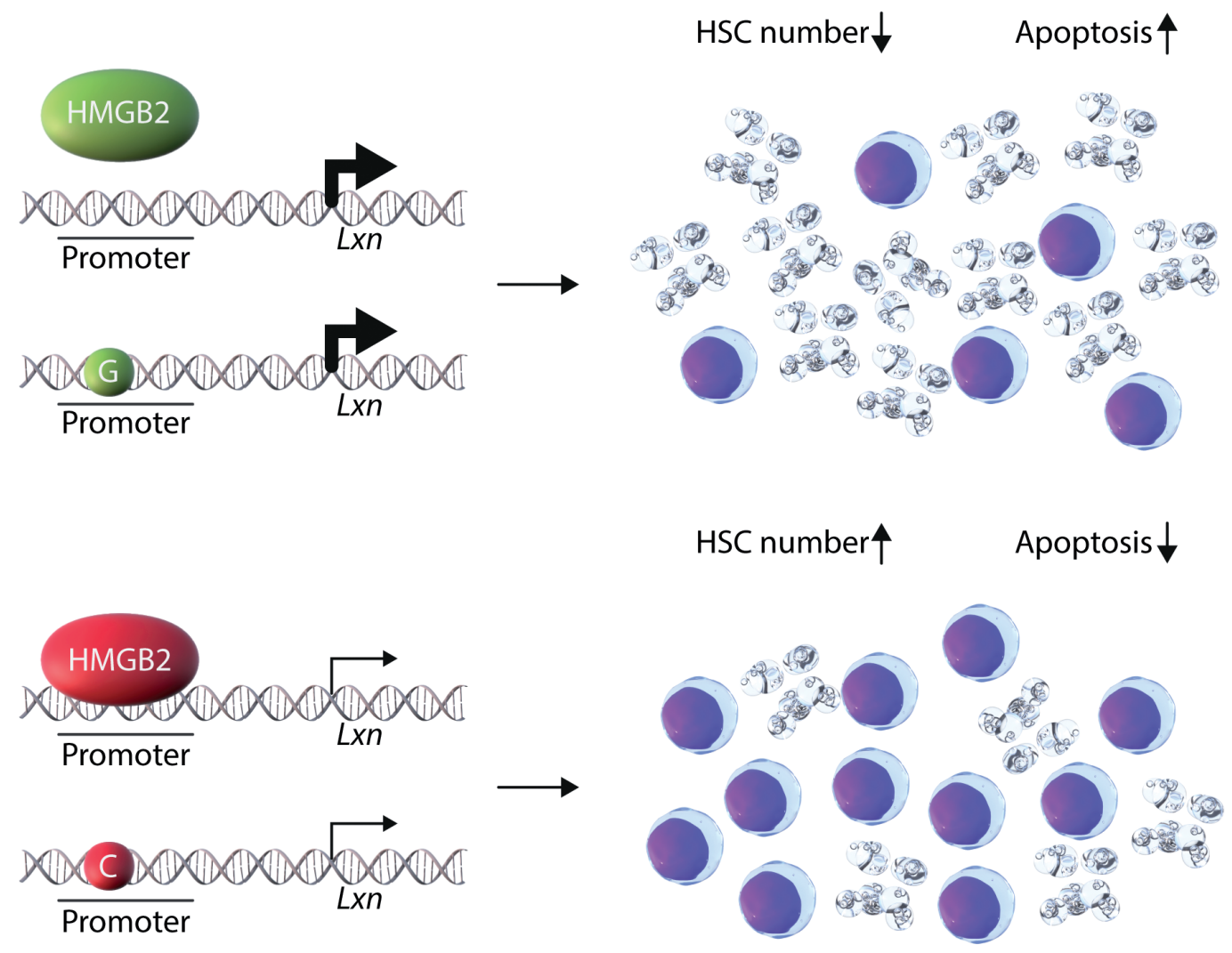

Figure 1. Cis- and trans-regulation of $L x n$ gene transcription modulates hematopoietic stem cell function. In trans-regulatory mechanisms of hematopoietic stem cell (HSC) function and number, HMGB2 protein binding to the $L x n$ gene promoter region positively regulates HSC by suppressing $L x n$ gene transcription and blocks apoptosis. In the cis-regulatory mechanism of HSC, the G allele in the SNP found in Lxn gene promoter region is associated with higher Lxn gene transcription and lower HSC number, whereas the $\mathrm{C}$ allele is associated with suppressed Lxn gene transcription and higher HSC number.

gene transcription may be regulated through epigenetic modification. HMGB2 is also known to regulate senescence-associated gene expression by orchestrating the chromatin landscape of the gene loci. ${ }^{16}$ As the authors discussed, it would be interesting to study the role of $L x n$ gene regulation by HMGB2 in the context of HSC senescence and aging. The SNP identified in this study was in the CG-rich region. Although direct evidence was not shown, these data imply a functional role of the genetic variation, such as SNP, in $L x n$ gene regulation via DNA methylation. Taken together, this report suggests epigenetic regulation of HSC via $L x n$ gene transcriptional regulation. The molecular mechanism of how epigenetic regulation by HMGB2 protein and genetic variation in the Lxn gene promoter region work together needs to be better understood.

This report is the first demonstration that genetic variation, especially SNP, is a determinant for the variations among different mouse strains in HSC pool size and function. The authors also discussed a similar observation made in humans, ${ }^{11}$ opening the possibility that the genetic variation in the $L x n$ gene promoter region may contribute to the pathogenesis of hematopoietic aplasia/neoplasia, such as bone marrow failure or leukemia. If epigenetic regulation of $L x n$ gene transcription is involved in these hematopoietic diseases, it could be a new therapeutic target for genetic correction/modification such as genome editing. Since other genes that regulate HSC function, besides the $L x n$ gene, could undergo genetic variation through cis-regulatory elements, it would be interesting to characterize SNP in the regulatory region of HSC regulatory genes. This would help to develop therapeutic strategies for personalized medicine.

\section{Acknowledgment}

This work was supported by KAKENHI from Japan Society for the Promotion of Science (JSPS) to TM (19K17833), and KAKENHI (17H05651, 18H02843), Princess Takamatsu Cancer Research Fund, MIRAI Research program and Center for Metabolic Regulation of Healthy Aging at Kumamoto University to $H T$.

\section{References}

1. Orkin SH, Zon LI. Hematopoiesis: an evolving paradigm for stem cell biology. Cell. 2008;132(4):631-644.

2. Gilad Y, Rifkin SA, Pritchard JK. Revealing the architecture of gene regulation: the promise of eQTL studies. Trends Genet. 2008;24(8): 408-415.

3. Wittkopp PJ, Kalay G. Cis-regulatory elements: molecular mechanisms and evolutionary processes underlying divergence. Nat Rev Genet. 2011;13(1):59-69

4. Beerman I, Rossi DJ. Epigenetic Control of Stem Cell Potential during Homeostasis, Aging, and Disease. Cell Stem Cell. 2015;16(6): 613-625

5. Henckaerts E, Langer JC, Snoeck HW. Quantitative genetic variation 
in the hematopoietic stem cell and progenitor cell compartment and in lifespan are closely linked at multiple loci in BXD recombinant inbred mice. Blood. 2004;104(2):374-379.

6. de Haan G, Nijhof W, Van Zant G. Mouse strain-dependent changes in frequency and proliferation of hematopoietic stem cells during aging: correlation between lifespan and cycling activity. Blood. 1997;89(5):1543-1550.

7. Geiger H, True JM, de Haan G, Van Zant G. Age- and stage-specific regulation patterns in the hematopoietic stem cell hierarchy. Blood. 2001;98(10):2966-2972.

8. de Haan G, Bystrykh LV, Weersing E, et al. A genetic and genomic analysis identifies a cluster of genes associated with hematopoietic cell turnover. Blood. 2002;100(6):2056-2062

9. Cahan P, Li Y, Izumi M, Graubert TA. The impact of copy number variation on local gene expression in mouse hematopoietic stem and progenitor cells. Nat Genet. 2009;41(4):430-437.

10. Liang Y, Jansen M, Aronow B, Geiger H, Van Zant G. The quantitative trait gene latexin influences the size of the hematopoietic stem cell population in mice. Nat Genet. 2007:39(2):178-188.

11. Zhang $C$, Fondufe-Mittendorf $Y N$, Wang $C$, et al. Latexin regulation by HMGB2 is required for hematopoietic stem cell maintenance. Haematologica. 2020;105(3):573-584

12. Ye ZJ, Kluger Y, Lian Z, Weissman SM. Two types of precursor cells in a multipotential hematopoietic cell line. Proc Natl Acad Sci U S A 2005;102(51):18461-18466.

13. You Y, Wen R, Pathak R, et al. Latexin sensitizes leukemogenic cells to gamma-irradiation-induced cell-cycle arrest and cell death through Rps3 pathway. Cell Death Dis. 2014;5:e1493.

14. Liu Y, Howard D, Rector K, et al. Latexin is down-regulated in hematopoietic malignancies and restoration of expression inhibits lymphoma growth. PLoS One. 2012;7(9):e44979.

15. Agresti A, Bianchi ME. HMGB proteins and gene expression. Curr Opin Genet Dev. 2003;13(2):170-178.

16. Aird KM, Iwasaki O, Kossenkov AV, et al. HMGB2 orchestrates the chromatin landscape of senescence-associated secretory phenotype gene loci. J Cell Biol. 2016;215(3):325-34.

\title{
Staying hydrated is important also for erythroblasts
}

\author{
Anupama Narla ${ }^{1}$ and Narla Mohandas ${ }^{2}$ \\ ${ }^{1}$ Department of Pediatrics, Stanford University, School of Medicine, Stanford, CA and ${ }^{2}$ Laboratory of Red Cell Physiology, New York \\ Blood Center, New York, NY, USA
}

E-mail: ANUPAMA NARLA - anunarla@stanford.edu or NARLAMOHANDAS - mnarla@nybc.org

doi:10.3324/haematol.2019.233999

I $\mathrm{n}$ this edition of Haematologica, Caulier and colleagues provide new insights into the role of PIEZO1, a mechanosensitive ion channel, in regulating normal human erythropoiesis. ${ }^{1}$ Defects in PIEZO1 have also been shown to lead to disordered erythropoiesis in hereditary xerocytosis, an inherited red cell disorder leading to red cell dehydration. ${ }^{2,3}$ Using in vitro cellular models of human erythropoiesis, the authors documented that the chemical activation of PIEZO1 either in an erythroid cell line model or in normal human hematopoietic stem cells (HSC) repressed erythroid differentiation. Importantly, they further documented that there was delayed erythroid differentiation in HSC from patients with PIEZO1 mutations. These findings provide unexpected and novel insights into the role of ion channels in the regulation of human erythropoiesis. ${ }^{1}$

Anemia is a significant health problem that affects nearly two billion people around the world. The major causes of anemia are: (i) an increased rate of destruction of circulating red cells in disorders that include red cell membrane disorders, sickle cell disease, immune hemolytic anemia, nutritional anemias and malaria; (ii) acute blood loss or splenic sequestration; and (iii) decreased production of red cells in the bone marrow due to ineffective erythropoiesis, which includes thalassemias, inherited bone marrow failure syndromes, infiltrative processes such as myelodysplastic syndrome and acute myeloid leukemia and suppression of erythropoiesis due to infection and medications. While significant progress has been made over the years to improve our understanding of the contribution of increased red cell destruction to anemia, much less is known about the extent of the effect of ineffective erythropoiesis and its contribution to anemia in the various red cell disorders. This is particularly true in the case of inherited red blood cell membrane disorders. The lack of progress has been due in part to a lack of an adequate and easily implementable methodology to study the complex process of human erythroid differentiation.

The generation of enucleated circulating human red cells is a complex biological process that begins in the bone marrow with the commitment of pluripotent HSC to the erythroid lineage (Figure 1). Subsequent stages of maturation include erythroid progenitors, burst-forming unit-erythroid and colony-forming unit-erythroid (CFUE), which can be identified by their development into representative clonal colonies of red cells in vitro. The CFU-E then undergoes terminal differentiation, progressing through four to five morphological stages, each having characteristic light microscopic and ultrastructural features. During terminal erythroid differentiation there is an increasing amount of hemoglobin synthesis accompanied by nuclear chromatin condensation and in the final stage of differentiation there is nuclear extrusion to generate an anucleate reticulocyte which over 2 to 3 days matures, first in the marrow and then in the circulation, into the discoid erythrocyte.

Significant progress has been made during the last decade in developing culture systems to study the differentiation of human CD34 cells intto enucleate reticulocytes and using various cell surface markers to monitor the progression through all stages of erythroid differentiation. ${ }^{4.7}$ These developments are enabling detailed characterization of normal and disordered human erythropoiesis. ${ }^{8-13}$ Importantly, as a result of this progress it is now possible to obtain insights into at what stage of the complex process of erythroid differentiation various genes contribute to ineffective erythropoiesis.

Using these in vitro cellular models of human erythropoiesis, the study by Caulier and colleagues documented that the chemical activation of PIEZO1 either in an ery- 